\title{
In the COVID-19 Era, Is It OK to Perform a Perfusion-Only SPECT/CT for the Diagnosis of Pulmonary Embolism?
}

\author{
Minseok Suh ${ }^{1}$
}

Received: 30 December 2021 / Revised: 19 January 2022 / Accepted: 27 January 2022 / Published online: 3 March 2022

(C) The Author(s), under exclusive licence to Korean Society of Nuclear Medicine 2022

Coronavirus disease 2019 (COVID-19) is a global and international public health emergency. The COVID-19 pandemic is having a profound impact on patient care and nuclear medicine practice, especially lung ventilation scans. There is still a lack of evidence whether the lung ventilation scan should be considered as an aerosol-generating procedure. However, since infection from COVID-19 is mainly transmitted by respiratory droplets and close contact, both test personnel and patients are at risk of transmission of COVID-19 during lung ventilation scans. In the inhalation procedure, the patient releases respiratory fluids in the form of droplets, which may carry the virus and transmit infection. For this reason, several recent papers suggest that a ventilation scan can be omitted and it can be replaced with perfusion-only single-photon emission tomography (SPECT)/computed tomography (CT) for the diagnosis of pulmonary embolism [1-3].

With the advent of hybrid SPECT/CT, it is possible to evaluate the pulmonary perfusion status in three-dimensional space, and when combined with the segmental anatomical information of each patient determined based on the CT scan, it offers a more accurate diagnosis [4]. Co-registered low-dose CT scans can directly assess the structural abnormality of the airways, lung parenchyma, and pleura, and areas with reduced perfusion without corresponding structural abnormalities are likely indicative of pulmonary embolism. Several studies have reported the diagnostic performance of perfusion-only SPECT/CT in the diagnosis of pulmonary thromboembolic disease [5-11] (Table 1). In common, perfusion-only SPECT/CT showed high sensitivity ranging from 88 to $100 \%$, and a high negative predictive value of $91-100 \%$. However, the specificity was reported to vary from as low as

Minseok Suh

mandu3710@gmail.com

1 Department of Nuclear Medicine, Seoul National University College of Medicine, Seoul National University Hospital, Seoul 03080, Republic of Korea
$51 \%$ to as high as $94 \%$. The study by Le Roux et al., which included the largest number of 393 patients, reported a specificity of $85 \%$ and a corresponding false-positive rate of $15 \%$. Overall, perfusion-only SPECT/CT showed a relatively high false-positive rate of around $20 \%$, so there is a possibility of overdiagnosis. The reason for the relatively high false-positive rate has not yet been well elucidated. Palmowski et al. hypothesized that vasoconstriction due to hypoxia can cause mild functional ventilatory disturbances present in ventilation SPECT but are morphologically invisible on low-dose CT [9].

The article published in this issue, "Utility of lung perfusion SPECT/CT in the detection of pulmonary thromboembolic disease: outcome analysis," also reports consistent results in pulmonary thromboembolic disease, including both pulmonary embolism and chronic thromboembolic pulmonary hypertension patients [12]. Based on a composite reference standard including clinical presentation, laboratory test results, other imaging test results, and follow-up data, it showed a sensitivity of $100 \%$ and a specificity of $78.6 \%$. In line with the previous studies, the false-positive rate was relatively high at $21.4 \%$.

Furthermore, a recent multicenter trial evaluated the role of lung scintigraphy in 145 COVID-19 patients, suspected of pulmonary embolism [13]. Compared with perfusion-only SPECT/CT, when ventilation/perfusion SPECT/CT was performed, an additional $31 \%$ of patients were able to rule out pulmonary embolism. Indeed, in the Spanish cohort, the standardized incidence of pulmonary embolism in the COVID-19 population was 310 cases per 100,000 person-years, which is significantly higher than that observed in the non-COVID-19 population (35 cases per 100,000 person-years) [14]. In hospitalized patients with COVID-19, the incidence of pulmonary embolism is reported to be as high as approximately 2.6-8.9\% [15]. Given the high incidence of pulmonary embolism in the COVID-19 population, the high false-positive rate of perfusion-only SPECT/CT has important implications.

Comprehensively, omitting ventilation scans will eventually lead to overdiagnosis of pulmonary thromboembolic 


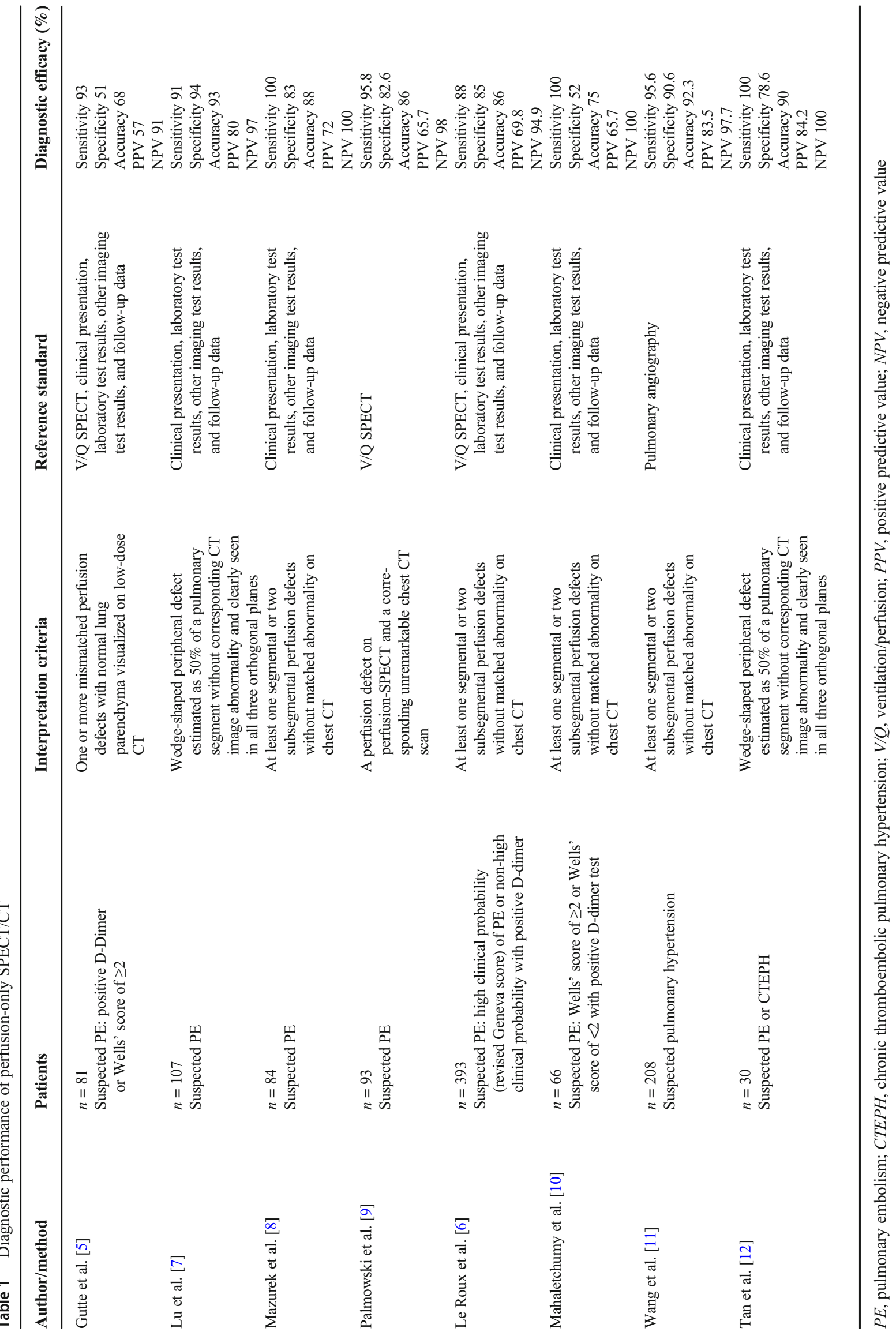


disease. Anticoagulation is the mainstay of treatment after the diagnosis of pulmonary embolism. Risks with continued use of anticoagulant therapy include bleeding, interactions with other drugs, repeated blood tests, and increased overall cost [16]. Diagnosis of pulmonary embolism also affects life insurance and medical procedures including preparation for surgery. Furthermore, it may lead to preoccupation with the patient, which can influence medical attention for similar symptoms in the future. In this context, the risk-benefit of performing perfusion-only SPECT/CT should be considered in the COVID-19 era.

There is no unified guideline for lung scintigraphy procedures to diagnose pulmonary embolism, and various strategies are recommended according to the circumstance of each medical facility, region, and country [17]. In line with Le Roux et al. [18], considering the risk-benefit of perfusion-only SPECT/CT omitting the ventilation scan cannot be justified, even in the COVID-19 situation. However, given the high sensitivity of perfusion-only SPECT/CT, it can be used in the pre-screening of patients, who are to be helped by ventilation scan. When ventilation scan is additionally performed only on subjects who have positive findings in perfusion-only SPECT/CT, both the transmission of COVID-19 and the risk of overdiagnosis can be minimized. Zuckier et al. have proposed a similar strategy to use stand-alone perfusion scintigraphy as a screening tool [19].

After the screening of eligible patients, preparations for protection must be made from the perspective of the patient, health care workers, and the environment. Patients should be pre-screened for symptoms with a questionnaire on arrival and checking body temperature, to verify if they are likely to have a COVID-19 infection. For the pulmonary function test (PFT), some countries recommend that the documented negative swab test result be presented $48-72 \mathrm{~h}$ prior to the test [20]; this can also be considered before the ventilation scan according to the circumstances. There are guidance documents for the protection of health care workers in the nuclear medicine procedure from COVID-19 infection [21-23]. Guidelines for PFT can also be referred to [20]. The guidelines commonly recommend wearing an appropriate filtering facepiece, and since there may be direct contact with respiratory droplets derived during the test, wearing a glove, visor, and gown is also recommended. In-between the procedure, the operator is recommended to stand next to the patient, facing the same direction, and to avoid face-to-face. Finally, after the examination, proper equipment cleaning and room ventilation are required. If more than one scan is to be performed in a row, timely reservations should be made to allow sufficient recirculation of the room $[20,22]$.

In the era of the COVID-19 pandemic (global pandemic), ventilation scan is recognized as a procedure that requires caution due to the potential for secretion of respiratory droplets containing viruses. It has been suggested that ventilation can be replaced by perfusion-only SPECT combined with CT giving information on structural abnormality. However, from a comprehensive perspective, perfusion-only SPECT/CT has the potential to increase the risk of overdiagnosis in pulmonary thromboembolic disease patients. Therefore, rather than replacing the ventilation scan with perfusion-only SPECT/CT, it should be used as a tool to pre-screen patients and if a ventilation scan is required, it is recommended to perform the procedure with appropriate preparation for protection.

Availability of Data and Material Not applicable

Author Contribution Conceptualization, investigation, writing - original draft, writing — review and editing: Minseok Suh.

\section{Compliance with Ethical Standards}

Conflict of Interest Minseok Suh declares no competing interests.

Ethical Approval All procedures performed in studies were in accordance with the ethical standards of the institutional and/or national research committee and with the 1964 Helsinki declaration and its later amendments or comparable ethical standards.

Informed Consent As an editorial article, obtaining informed consent was waived.

Consent for Publication None

\section{References}

1. Burger IA, Niemann T, Patriki D, Fontana F, Beer JH. Is there a role for lung perfusion [(99m)Tc]-MAA SPECT/CT to rule out pulmonary embolism in COVID-19 patients with contraindications for iodine contrast? Eur J Nucl Med Mol Imaging. 2020;47:2062-3.

2. $\mathrm{Lu} \mathrm{Y}$, Macapinlac HA. Perfusion SPECT/CT to diagnose pulmonary embolism during COVID-19 pandemic. Eur J Nucl Med Mol Imaging. 2020;47:2064-5.

3. Tulchinsky M, Osmany S. The American College of Nuclear Medicine Guidance on operating procedures for a nuclear medicine facility during COVID-19 pandemic. Clin Nucl Med. 2021;46: $571-4$.

4. Suh M, Kang YK, Ha S, Kim YI, Paeng JC, Cheon GJ, et al. Comparison of two different segmentation methods on planar lung perfusion scan with reference to quantitative value on SPECT/CT. Nucl Med Mol Imaging. 2017;51:161-8.

5. Gutte H, Mortensen J, Jensen CV, Johnbeck CB, von der Recke P, Petersen CL, et al. Detection of pulmonary embolism with combined ventilation-perfusion SPECT and low-dose CT: head-to-head comparison with multidetector CT angiography. J Nucl Med. 2009;50:1987-92.

6. Le Roux PY, Robin P, Delluc A, Abgral R, Palard X, Tissot V, et al. Additional value of combining low-dose computed tomography to V/Q SPECT on a hybrid SPECT-CT camera for pulmonary embolism diagnosis. Nucl Med Commun. 2015;36:922-30.

7. Lu Y, Lorenzoni A, Fox JJ, Rademaker J, Vander Els N, Grewal RK, et al. Noncontrast perfusion single-photon emission CT/CT scanning: a new test for the expedited, high-accuracy diagnosis of acute pulmonary embolism. Chest. 2014;145:1079-88. 
8. Mazurek A, Dziuk M, Witkowska-Patena E, Piszczek S, Gizewska A. The utility of hybrid SPECT/CT lung perfusion scintigraphy in pulmonary embolism diagnosis. Respiration. 2015;90:393-401.

9. Palmowski K, Oltmanns U, Kreuter M, Mottaghy FM, Palmowski M, Behrendt FF. Diagnosis of pulmonary embolism: conventional ventilation/perfusion SPECT is superior to the combination of perfusion SPECT and nonenhanced CT. Respiration. 2014;88:291-7.

10. Thanuja M, Maimanah M, Sara U. Diagnosis of pulmonary embolism: a comparison between ventilation/perfusion SPECT/CT and perfusion-only SPECT/CT. Med J Malaysia. 2020;75:490-3.

11. Wang L, Wang M, Yang T, Wu D, Xiong C, Fang W. A prospective, comparative study of ventilation-perfusion planar imaging and ventilation-perfusion SPECT for chronic thromboembolic pulmonary hypertension. J Nucl Med. 2020;61:1832-8.

12. Tan T-H. Utility of lung perfusion SPECT/CT in detection of pulmonary thromboembolic disease: outcome analysis. Nucl Med Mol Imaging. 2022.

13. Le Roux PY, Bonnefoy PB, Bahloul A, Denizot B, Barres B, Moreau-Triby $\mathrm{C}$, et al. Lung scintigraphy for pulmonary embolism diagnosis in COVID-19 patients: a multicenter study. J Nucl Med. 2021. https://doi.org/10.2967/jnumed.121.262955.

14. Miro O, Jimenez S, Mebazaa A, Freund Y, Burillo-Putze G, Martin A, et al. Pulmonary embolism in patients with COVID-19: incidence, risk factors, clinical characteristics, and outcome. Eur Heart J. 2021;42:3127-42.

15. Sakr Y, Giovini M, Leone M, Pizzilli G, Kortgen A, Bauer M, et al. Pulmonary embolism in patients with coronavirus disease-2019 (COVID-19) pneumonia: a narrative review. Ann Intensive Care. 2020;10:124.
16. Hutchinson BD, Navin P, Marom EM, Truong MT, Bruzzi JF. Overdiagnosis of pulmonary embolism by pulmonary CT angiography. AJR Am J Roentgenol. 2015;205:271-7.

17. Zuckier LS. To everything there is a season: taxonomy of approaches to the performance of lung scintigraphy in the era of COVID-19. Eur J Nucl Med Mol Imaging. 2021;48:666-9.

18. Le Roux PY, Le Gal G, Salaun PY. Lung scintigraphy for pulmonary embolism diagnosis during the COVID-19 pandemic: does the benefit-risk ratio really justify omitting the ventilation study? Eur J Nucl Med Mol Imaging. 2020;47:2499-500.

19. Zuckier LS, Moadel RM, Haramati LB, Freeman LM. Diagnostic evaluation of pulmonary embolism during the COVID-19 pandemic. J Nucl Med. 2020;61:630-1.

20. Crimi C, Impellizzeri P, Campisi R, Nolasco S, Spanevello A, Crimi N. Practical considerations for spirometry during the COVID-19 outbreak: literature review and insights. Pulmonology. 2021;27:438-47.

21. SNMMI Statement: COVID-19 and ventilation/perfusion (V/Q) lung studies. J Nucl Med Technol. 2021;49:12A.

22. Buscombe JR, Notghi A, Croasdale J, Pandit M, O'Brien J, Graham $\mathrm{R}$, et al. COVID-19: guidance for infection prevention and control in nuclear medicine. Nucl Med Commun. 2020;41:499-504.

23. Bang JI, Lee HY, Cho YS, Choi H, Chong A, Eo JS, et al. $\mathrm{KSNM} / \mathrm{KSID} / \mathrm{KOSHIC}$ guidance for nuclear medicine department against the coronavirus disease 2019 (COVID-19) pandemic. Nucl Med Mol Imaging. 2020;54:163-7.

Publisher's Note Springer Nature remains neutral with regard to jurisdictional claims in published maps and institutional affiliations. 